\title{
TÁC ĐộNG CỦA ĐẶC ĐIỂM TÍNH CÁCH BIG - FIVE VÀ LÃNH ĐẠO ĐẠO ĐỨC ĐẾN SỰ HÀI LÒNG TRONG CÔNG VIỆC, NGHIÊN CÚU TRƯỜNG HỢP TRONG CÁC HỆ THỐNG SIÊU THỊ LỚN TẠI THÀNH PHỐ HỒ CHÍ MINH
}

\author{
NGUYẼ̃N THÀNH LONG ${ }^{1}$, TRẦN THỊ MINH NHU² \\ ${ }^{1}$ Trường Đại học Công nghiệp Tp. HCM, \\ ${ }^{2}$ Truờng Đại hoc Kinh Tế Tp. HCM \\ nguyenthanhlong@iuh.edu.vn
}

Tóm tắt. Nghiên cứu này nhằm xác định tác động của đặc điểm tính cách Big-Five và lãnh đạo đạo đức tới sự hài lòng trong công việc, nghiên cứu trường hợp trong các hệ thống siêu thị lớn tại $\mathrm{Tp}$. $\mathrm{HCM}$. Kết hợp cả hai phương pháp nghiên cứu định tính và định lượng, tác giả tiến hành khảo sát 380 đối tượng là nhân viên, quản lý làm việc tại các siêu thị lớn ở Tp. HCM. Kết quả nghiên cứu cho thấy, các đặc điểm tính cách có tác động đến lãnh đạo đạo đức và sự hài lòng trong công việc. Thư nhất, ba yếu tố của đặc điểm tính cách như sẵn sàng trải nghiệm, tận tâm và dễ chịu có ảnh hưởng tích cực đến lãnh đạo đức. Thứ hai, ba yếu tố sẵn sàng trải nghiệm, tận tâm và hướng ngoại có ảnh hưởng tính cực đến sự hài lòng trong công việc. Thứ $b a$, riêng yếu tố tâm lý bất ổn có ảnh hưởng tiêu cực đến cả lãnh đạo đạo đức và sự hài lòng trong công việc. Từ kết quả này, nghiên cứu đưa ra một số hàm ý quản trị nhằm nhằm gợi ý cho nhà lãnh đạo quản lý và nhà tuyển dụng nắm bắt được đặc điểm tính cách của nhân viên nhằm đáp ứng mang lại sự hài lòng trong công việc để đạt được kết quả làm việc tốt nhất.

Từ khóa. Năm đặc điểm tính cách Big - Five, lãnh đạo đạo đức và sự hài lòng

\section{IMPACT OF BIG FIVE PERSONALITY TRAITS AND ETHICAL LEADERSHIP ON JOB SATISFACTION, A CASE STUDY AT LARGE SUPERMARKETS IN HO CHI MINH CITY}

\begin{abstract}
This study aimed to determine the impact of Big-Five personality traits and ethical leadership on job satisfaction, the case study of large supermarkets in Ho Chi Minh City. Combining both quatitative and quanlitative research methods, this study used self completed questionnaires to investigate 380 employees and managers, who have worked at large supermarkets in Ho Chi Minh City. The research results show that personality traits have an impact on ethical leadership and job satisfaction. First, the three factors of personality traits such as openness to experience, conscientiousness and agreeableness have positive effect on ethical leadership. Second, openness to experience, conscientiousness and extraversion are found to be important antecedents for job satisfaction. Third, neuroticism has negative effect on both moral leadership and job satisfaction. Fourth, results did not show any significant relationship between extraversion and ethical leadership; between agreeableness and job satisfaction. Based on the findings, the study provides management implications of leaders and human resources managers perceptions of the personality traits of employees in response to job satisfaction to achieve the best results. A summary of the overall findings of the study are discussed as well as future directions for further study.
\end{abstract}

Keywords. Big-Five personality traits; ethical leadership and job satisfaction. 


\section{GIỚI THIÊU}

Để trở thành một nhà lãnh đạo thành công ngoài trí tuệ, tố chất và kỹ năng thì đạo đức cũng là yếu tố rất quan trọng. Một nhà lãnh đạo có đạo đức sẽ được nhân viên kính trọng và gắn bó lâu dài với công ty, đem lại sự hài lòng trong công việc cho nhân viên. Các nhà nghiên cứu về hành vi tổ chức cho thấy, đặc điểm tính cách cá nhân ảnh hưởng nhiều đến sự hài lòng trong công việc của nhân viên. Do đó, vấn đề về đặc điểm tính cách phải được quan tâm nhiều hơn nữa trong việc tuyển dụng và đào tạo, bố trí công việc, tạo điều kiện và môi trường làm việc tốt sẽ giúp nhân viên gắn bó lâu dài hơn với tổ chức, đem lại sự hài lòng trong công việc cho nhân viên.

Thị trường bán lẻ Việt Nam đã trải qua nhiều biến động trong quá trình hội nhập, với sự góp mặt của nhiều thương hiệu lớn và mang tính cạnh tranh cao. Để có thể cạnh tranh và đứng vững trên thị trường các nhà đầu tư hệ thống siêu thị lớn cần trang bị cho mình quy trình làm việc hiện đại kết hợp sự linh hoạt trong nắm bắt được tâm lý khách hàng mà đặt biệt là biết cách quản lý nhân viên nhằm mang lại sự hài lòng trong công việc cho họ. Để làm được điều đó đòi hỏi các nhà quản lý siêu thị cần phải có đạo đức với bên trong và trách nhiệm xã hội với cộng đồng. Vì vậy để nhân viên có thể làm việc gẳng bó lâu dài với tổ chức cần quan tâm đến đặc điểm tính cách của nhân viên để phân bổ nguồn lực hợp lý làm cho tổ chức ngày một phát triển hơn.

\section{CƠ SỞ LÝ THUYẾT VÀ MÔ HÌNH NGHIÊN CỨU}

\subsection{Cơ sở lý thuyết}

\section{* Năm đặc điểm tính cách Big - Five}

Tính cách được định nghĩa là tập hợp các hành vi, nhận thức và các mẫu cảm xúc đặc trưng phát triển từ các yếu tố sinh học và môi trường [1]. Tính cách thường được chia thành các yếu tố được xác định theo thống kê được gọi là Big Five, đó là tính sẵn sàng trải nghiệm, tận tâm, hướng ngoại, dễ chịu và tâm lý bất ổn.

Mô hình nhân cách Big Five dựa trên những phát hiện từ một số nhà nghiên cứu độc lập trong những năm 1950. Theo McCrae và John (1992) [2], Big Five là tên gọi chung của năm đặc điểm lớn của tính cách, bao gồm: hướng ngoại (Extraverson), sự dễ chịu (Agreeableness), tận tâm (Conscientiousness), tâm lý bất ổn (Neuroticism) và sẵn sàng trải nghiệm (Openness to Experience).

\section{* Lãnh đạo đạo đức}

Theo nghiên cứu của Brown, Treviño, và Harrison (2005) [3], lãnh đạo đạo đức được định nghĩa là diễn giải chuẩn mực hành vi thông thường thông qua các hành động cá nhân và mối quan hệ giữa các cá nhân và thúc đẩy hành vi của nhân viên thông qua giao tiếp hai chiều, củng cố và ra quyết định. Các nhà lãnh đạo đạo đức đã được mô tả là cả những người đạo đức và các nhà quản lý đạo đức. Họ thường được mô tả là quan tâm và thực sự quan tâm đến nhân viên của họ và luôn trung thực, đáng tin cậy, nguyên tắc, thuyết phục. Đây là hình thức lãnh đạo đã được xác định từ quan điểm của xã hội học, lãnh đạo dựa trên các quy tắc chuẩn mực và giá trị mà được thể hiện thông qua việc ứng xử, hành động, ra quyết định, trừng phạt và khen thưởng. Lãnh đạo đạo đức được thể hiện qua các khía cạnh như: mối liên hệ giữa lãnh đạo đạo đức và lãnh đạo chiến lược [4]; lòng tự trọng và đặc tính [5]; tính cách [6]; hành vi sáng tạo [7].

\section{* Sụ hài lòng trong công việc}

Theo Hoppock và Splegler (1935) [8] sự hài lòng trong công việc được xem như bất kỳ sự kết hợp nào về hoàn cảnh tâm lý, sinh lý và môi trường khiến một người trung thực nói rằng tôi hài lòng với công việc của tôi. Còn theo Locke (1976) [9] đã xác định sự hài lòng trong công việc là trạng thái cảm xúc thú vị hoặc tích cực từ kết quả thẩm định công việc hoặc kinh nghiệm làm việc, bao gồm một tập hợp các yếu tố tạo ra cảm giác hài lòng. Sự hài lòng trong công việc thường thiên về trạng thái cảm xúc hoặc tinh thần của một con người, nó cho thấy mức độ yêu thích và bận tâm của nhân viên đối với công việc. Các yếu tố thường tạo nên sự hài lòng cho nhân viên như bản chất của công việc, tiền lương, căng thẳng trong công việc, môi trường làm việc, đồng nghiệp, người giám sát và giờ làm việc,,..

Theo Greenberg và Baron (2008) [10], sự hài lòng trong công việc là phản ứng nhận thức, tình cảm và đánh giá của nhân viên đối với công việc của họ. Còn theo Pitaloka và Sofia (2014) [11] thì sự hài lòng trong công việc là cảm giác tâm lý và cảm xúc đối với công việc của mình. Sự hài lòng công việc được đo 
lường thông qua các chỉ số sau đây: công việc tự nó, lợi ích, mối quan hệ với các nhân viên khác, cơ hội quảng cáo.

Nhìn chung, sự hài lòng trong công việc sẽ mang đến nhiều lợi ích cho cả nhân viên và tổ chức. Từ quan điểm của nhân viên, sự hài lòng trong công việc thường dẫn đến hạnh phúc, thoải mái và tận hưởng những gì họ đang làm. Từ quan điểm tổ chức, sự hài lòng công việc của nhân viên dẫn đến hiệu quả trong kinh doanh sẽ tốt hơn. Ngoài ra, nhân viên hài lòng với công việc thường có xu hướng sáng tạo hơn, hỗ trợ hơn cho các nhà quản lý trong việc ra quyết định [12].

\subsection{Mô hình nghiên cứu}

Theo McCrae và John (1992) [2], Big Five là tên gọi chung của năm đặc điểm lớn của tính cách, bao gồm: hướng ngoại, sự dễ chịu, tận tâm, tâm lý bất ổn và sẵn sàng trải nghiệm.

\section{* Huớng ngoại}

Hướng ngoại bao gồm các đặc điểm như tính xã hội, như quyết đoán, cởi mở và hòa đồng. Những người có điểm "E - Extraverson" cao thường hòa đồng, quyết đoán, năng động, táo bạo, tràn đầy năng lượng, phiêu lưu và biểu cảm. Họ có xu hướng tìm kiếm cơ hội để tương tác với mọi người, thích giao du, nói nhiều, thân thiện, năng động và dễ hành động trước suy nghĩ.

\section{* Sưdễ chịu}

Theo Digman (1990) [13], những đặc điểm liên quan đến tính dễ chịu là sự khiêm tốn và lòng vị tha. Những người có điểm "A - Agreeableness" cao thường được yêu thích, tôn trọng, tốt bụng, dễ tin tưởng và giúp đỡ người khác. Họ có thể có ít kẻ thù, biết thông cảm, chia sẻ với bạn bè và người thân của họ, cũng như với người lạ.

\section{* Tận tâm}

Tận tâm là một đặc điểm có thể được mô tả như một xu hướng chịu trách nhiệm, đáng tin cậy, kiên trì, kỹ lưỡng, có thể kiểm soát, đúng giờ, làm việc chăm chỉ và có lập kế hoạch rõ ràng,.. Những người có điểm "C - Conscientiousness" cao có khả năng thành công trong học tập và sự nghiệp. Họ là những người sống quy tắc, cẩn thận và có trách nhiệm.

\section{* Tâm lý bất ổn}

Tâm lý bất ổn là đặc điểm được mô tả như một sự lo lắng và không ổn định. Những người có điểm "N - Neuroticism" cao thường là những người hay nhạy cảm, buồn rầu, lo lắng và và đôi khi hoang tưởng. Họ hay giận dữ hoặc dễ dàng tức giận, có xu hướng tự phê phán và không chắc chắn về bản thân.

\section{* Sẵn sàng trải nghiệm}

Sẵn sàng trải nghiệm là tính cách mạnh mẽ, thông minh, sáng tạo và thích khám phá. Những người có điểm "O - Openness to Experience" cao được đặc trưng bởi trí tưởng tượng cao là người yêu thích học tập, thích nghệ thuật, thích tham gia vào một ngành nghề hoặc sở thích sáng tạo và thích gặp gỡ những người mới. Họ là kiểu người sáng tạo, hóm hỉnh, muốn tìm hiểu những điều mới mẻ, và có sở thích đa dạng.

Nghiên cứu này thực hiện đo lường tác động của các đặc điểm tính cách Big-Five và lãnh đạo đạo đức đến sự hài lòng trong công việc của nhân viên siêu thị. Trên cơ sở tiếp cận lý thuyết từ các công trình nghiên cứu trên, tác giả đưa ra định hướng nghiên cứu thông qua kê thừa các nghiên cứu: Özbağ (2016), Boakye và Gyambrah (2017), Naiyananont và Smuthranond (2016), Yang và Hwang (2014) và Bui (2017). Trên cơ đó, tác giả đề xuất mô hình cho nghiên cứu như sau: 


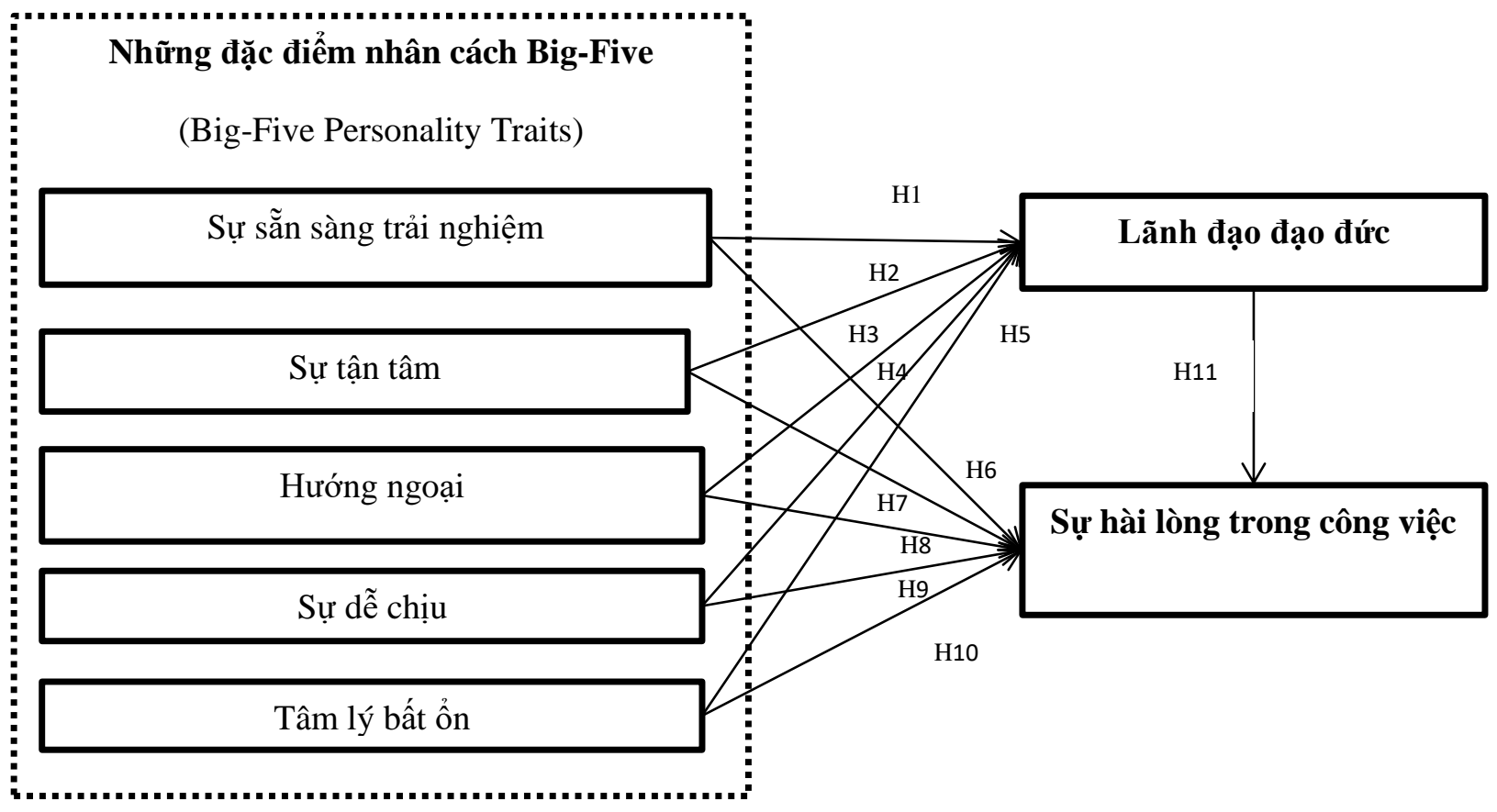

Hình 1: Mô hình nghiên cứu đề xuất

\section{* Đặc điểm năm nhân cách (Big-Five) và lãnh đạo đạo đức}

Một số nghiên cứu đã liên kết các đặc điểm nhân cách với hành vi lãnh đạo đạo đức, cung cấp một cơ sở thực nghiệm cho lập luận rằng các đặc điểm nhân cách có ý nghĩa quan trọng với lãnh đạo đạo đức [19]; (Özbağ, 2016), (Boakye và Gyambrah, 2017). Những nghiên cứu này nhằm mục đích xác định mối quan hệ giữa những đặc điểm tính cách của Big-Five và sự lãnh đạo đạo đức. Trên cơ sở đó, tác giả đề xuất các giả thuyết sau đây:

H1: Sụ sã̃n sàng trải nghiệm có ảnh hưởng tích cưcc (+) đến lãnh đạo đạo đức

H2: Sự tận tâm có ảnh hưởng tích cực (+) đến lãnh đạo đạo đức.

H3: Hướng ngoại có ảnh huởng tích cục (+) đến lãnh đạo đạo đức.

H4: Sự dễ chịu có ảnh hưởng tích cực (+) đến lãnh đạo đạo đức.

H5: Tâm lý bất ổn sẽ có ảnh hưởng tiêu cực (+) đến lãnh đạo đạo đức

\section{* Đặc điểm năm nhân cách (Big-Five) và sụ hài lòng trong công việc}

Có nhiều nghiên cứu giải thích mối quan hệ giữa đặc điểm tính cách Big-Five và sự hài lòng trong công việc như nghiên cứu của Bruk-Lee, Khoury, Nixon, Goh, và Spector (2009); Foulkrod, Field và Brown (2010); Jones, Hill và Henn (2015); Van den Berg và Feij (2003); Zhai, Willis, O'Shea, Zhai, và Yang (2013) [20]-[24]. Các tác giả này đã nghiên cứu mối quan hệ giữa các đặc điểm nhân cách cá nhân và sự hài lòng trong công việc. Trên cơ sở đó, tác giả đề xuất các giả thuyết sau đây:

H6: Sụ sẵn sàng trải nghiệm có ảnh huởng tích cưc đến sụ hài lòng trong công việc.

H7: Sụ tận tâm có ảnh hưởng tích cưc đến sụ hài lòng trong công việc.

H8: Hướng ngoại có ảnh hưởng tích cực đến sự hài lòng trong công việc.

H9: Sư dễ chịu có ảnh huơơng tích cực đến sự hài lòng trong công việc.

H10: Tâm lý bất ổn sẽ có ảnh hưởng tiêu cực đến sụ hài lòng trong công việc.

\section{* Lãnh đạo đạo đức và sụ hài lòng}

Neubert, Carlson, Kacmar, Roberts, và Chonko (2009; Yates (2014) [25], [26] đã củng cố khái niệm lý thuyết nâng cao nhận thức về lãnh đạo đạo đức làm tăng sự hài lòng công việc. Hai nghiên cứu này cho thấy mối quan hệ giữa lãnh đạo đức và sự hài lòng của nhân viên. Trên cơ sở đó, tác giả đề xuất các giả thuyết sau đây:

H11: Lãnh đạo đạo đức có ảnh hưởng tích cực đến sụ hài lòng trong công việc 


\section{PHƯƠNG PHÁP GHIÊN CÚU}

Nghiên cứu này được thực hiện thông qua 3 giai đoạn:

Giai đoạn 1, nội dung giai đoạn này tác giả sử dụng phương pháp chuyên gia, trên cơ sở tham khảo ý kiến chuyên gia và thảo luận nhóm nhằm hoàn thiện thang đo và thiết kế bảng câu hỏi điều tra.

Giai đoạn 2, nội dung giai đoạn này sẽ thực hiện: Kiểm định độ tin cậy của thang đo với hệ số Cronbach's Alpha và phân tích nhân tố khám phá. Theo Hair và cộng sự (2006) [27] kích thước mẫu tối thiểu phải $\geq \mathrm{m} \times 5$, trong đó $\mathrm{m}$ là số lượng biến quan sát. Vậy, với 38 biến quan sát trong nghiên cứu này kích thước mẫu tối thiểu phải $\geq 190$. Tuy nhiên, để đảm bảo độ tin cậy cao, tác giả tiến hành khảo sát 460 đối tượng là nhân viên đang làm việc trong các siêu thị lớn tại Tp. HCM. Tất cả số liệu thu thập từ bảng câu hỏi điều tra được mã hóa, xử lý bằng phần mềm SPSS và AMOS. Theo Nunnally và Bernstein (1994) [28], những biến quan sát nào có hệ số tương quan biến tổng lớn hơn 0,3 và có hệ số Cronbach's Alpha lớn hơn 0,7 thì mới đảm bảo độ tin cậy của thang đo. Mục đích của phân tích nhân tố khám phá để thu nhỏ và tóm tắt các dữ liệu. Phương pháp này dựa vào tỷ số rút trích nhân tố (Eigenvalue), phân tích nhân tố là thích hợp và các biến quan sát trong tổng thể có mối tương quan với nhau khi tổng phương sai trích phải > 50\%, hệ số KMO nằm trong khoảng 0,5 đến 1 , hệ số Sig. $\leq 5 \%$, các giá trị Factor loading của tất cả các biến quan sát đều $>0,5 ;$ chênh lệch trọng số $\lambda \mathrm{iA}-\lambda \mathrm{iB}$ đều $>0,3$.

Giai đoan 3, hai nội dung trong giai đoạn này, (1) phân tích nhân tố khẳng định CFA và (2) Kiểm định mô hình nghiên cứu bằng phân tích mô hình cấu trúc tuyến tính SEM (Structural Equation Modeling). Mục đích của phân tích nhân tố khẳng định CFA giúp làm sáng tỏ: (1) Tính đơn hướng; (2) Độ tin cậy của thang đo; (3) Giá trị hội tụ; (4) Giá trị phân biệt. Theo Hair và cộng sự (1998) [29]; Steenkamp và Van Trijp (1991) [30], một mô hình nghiên cứu được xem là phù hợp với dữ liệu thị trường nếu kiểm định Chi-quare có giá trị $\mathrm{P}$-value $<5 \% ; \mathrm{CMIN} / \mathrm{df} \leq 2$, một số trường hợp CMIN/df có thể $\leq 3$ [31]; GFI, TLI, CFI $\geq 0,9$. Tuy nhiên, theo quan điểm gần đây của các nhà nghiên cứu thì GFI vẫn có thể chấp nhận được khi lớn hơn 0,$8 ;$ RMSEA $\leq 0,08$. Ngoài những chỉ tiêu trên, kết quả kiểm định cũng phải đảm bảo độ tin cậy tổng hợp $>0,6$; phương sai trích phải lớn hơn 0,5 [29].

\section{KẾT QUẢ NGHIÊN CÚ̉U}

\subsection{Thông kê mô tả}

Tổng số phiếu khảo sát phát ra là 460, Tổng số phiếu thu về là 414 phiếu, trong đó có 34 phiếu bị loại do trả lời không đầy đủ hoặc không trả lời. Như vậy, chỉ có 380 phiếu khảo sát trả lời hợp lệ được sử dụng trong phân tích dữ liệu. Trong số 380 quan sát hợp lệ, có 200 nữ, chiếm 52,6\%; 180 nam chiếm tỷ lệ 47,8\%.

\subsection{Kiểm tra độ tin cậy của thang đo}

Kết quả kiểm tra độ tin cậy Cronbach's Alpha cho thấy có 4 biến quan sát không đảm bảo độ tin cậy sẽ bị loại, bao gồm: A4, EL2, EL6, JS6 vì có hệ số tương quan biến tổng nhỏ hơn 0,3. Như vậy, bộ thang đo đưa vào phân tích nhân tố khám phá EFA còn lại 34 biến quan sát.

\subsection{Phân tích nhân tố khám phá EFA (Exploratory Factor Analysis)}

Kết quả phân tích nhân tố cho thấy, các biến được trích thành 7 nhóm, với tổng phương sai trích (hay tổng biến thiên được giải thích) bằng $69,01 \%>50 \%$; hệ số $\mathrm{KMO}=0,933$ nằm trong khoảng $0,5 \leq \mathrm{KMO} \leq$ 1; vì vậy, phân tích nhân tố (EFA) là thích hợp. Kiểm định Bartlett với Sig.=.000, thể hiện mức ý nghĩa cao. Tất cả giá trị Factor loading của các biến quan sát đều lớn hơn 0,5 ; chênh lệch trọng số $\lambda_{\mathrm{i}} \mathrm{A}-\lambda_{\mathrm{i}} \mathrm{B}$ đều lớn hơn 0,3 nên được chấp nhận. 
Bảng 1: Kết quả xoay nhân tố

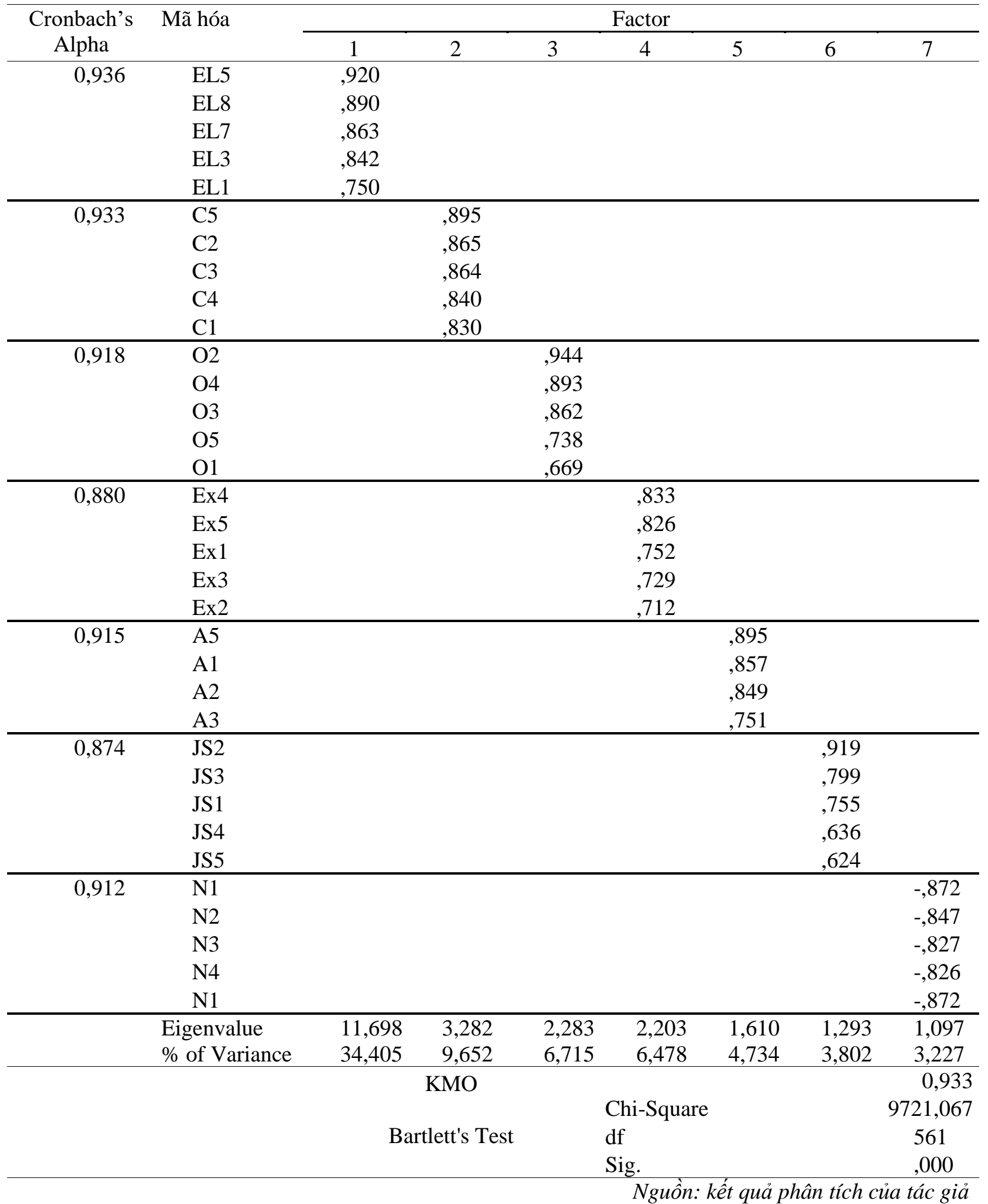




\subsection{Phân tích nhân tố khẳng định (CFA)}

Kết quả phân tích CFA của mô hình đo lường cuối cùng cho thấy, Chi-square $=697,930, \mathrm{df}=506, \mathrm{P}$ $=.000$, Chi-square $/ \mathrm{df}=1,379<3 ; \mathrm{GFI}=.904, \mathrm{TLI}=.978$ và $\mathrm{CFI}=.980$ lớn hơn $0.8 ; \mathrm{RMSEA}=0,032$ $<0,05$ mô hình là hợp lệ và đảm bảo độ tin cậy (chi tiết theo hình 2).

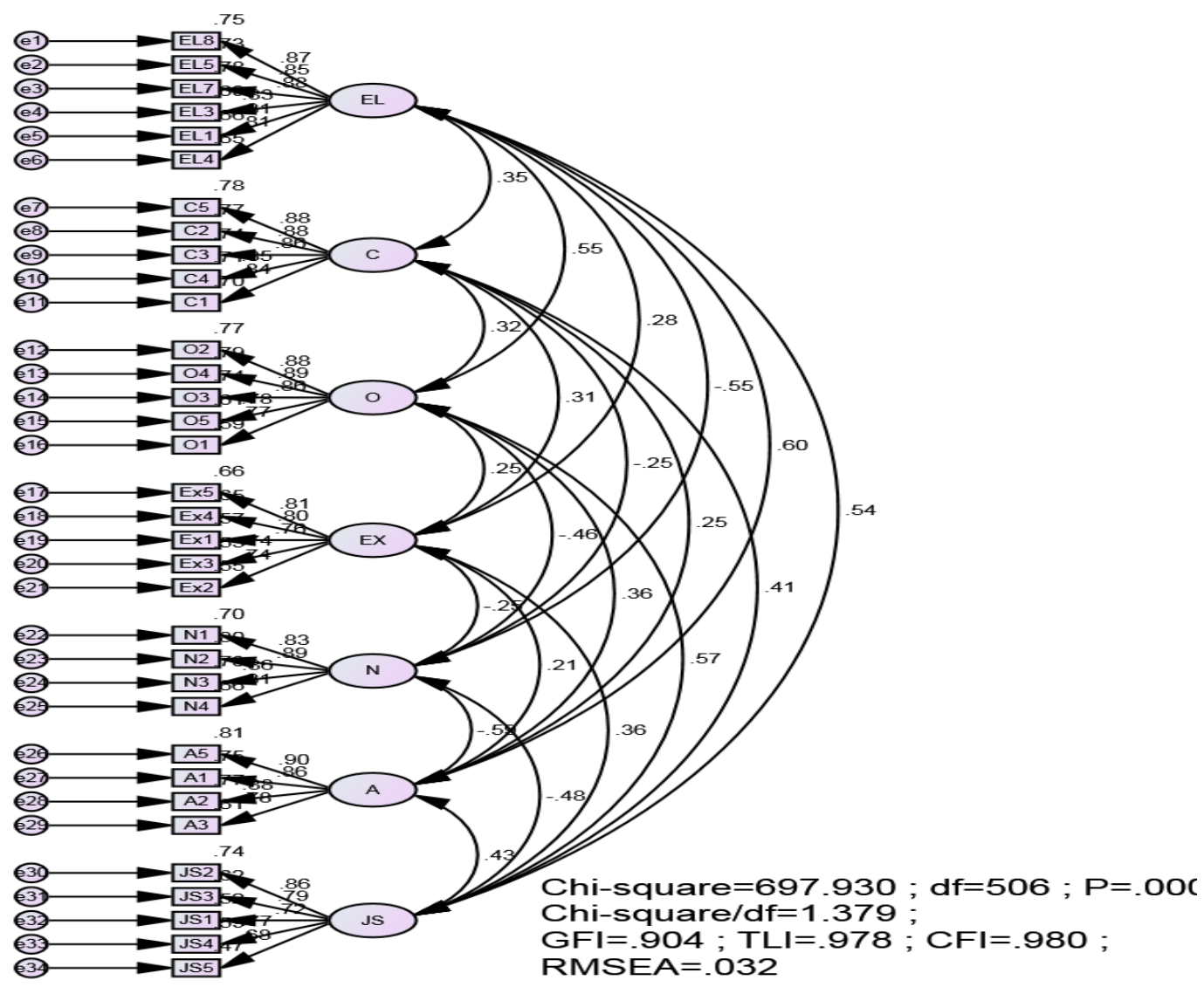

Hình 2: Phân tích nhân tố khẳng định CFA

\subsection{Phân tích mô hình cấu trúc tuyến tính SEM (Structural Equation Modeling)}

\subsubsection{Kiểm định mức độ phù hợp của mô hình}

Kết quả ước lượng (chuẩn hóa) mô hình nghiên cứu cho thấy, mô hình là phù hợp với dữ liệu, chi bình phương $=697,930 ;$ có bậc tự do $=506$, giá trị $\mathrm{P}=, 000 ; \mathrm{CMIN} / \mathrm{df}=1,379<3$. Các chỉ tiêu đo lường $\mathrm{GFI}=$ 0,$904 ; \mathrm{TLI}=0,978 ; \mathrm{CFI}=0,9806$ đều lớn hơn 0,$8 ; \mathrm{RMSEA}=0,032<0.08$ đều đạt yêu cầu (Chi tiết theo Hình 3). 


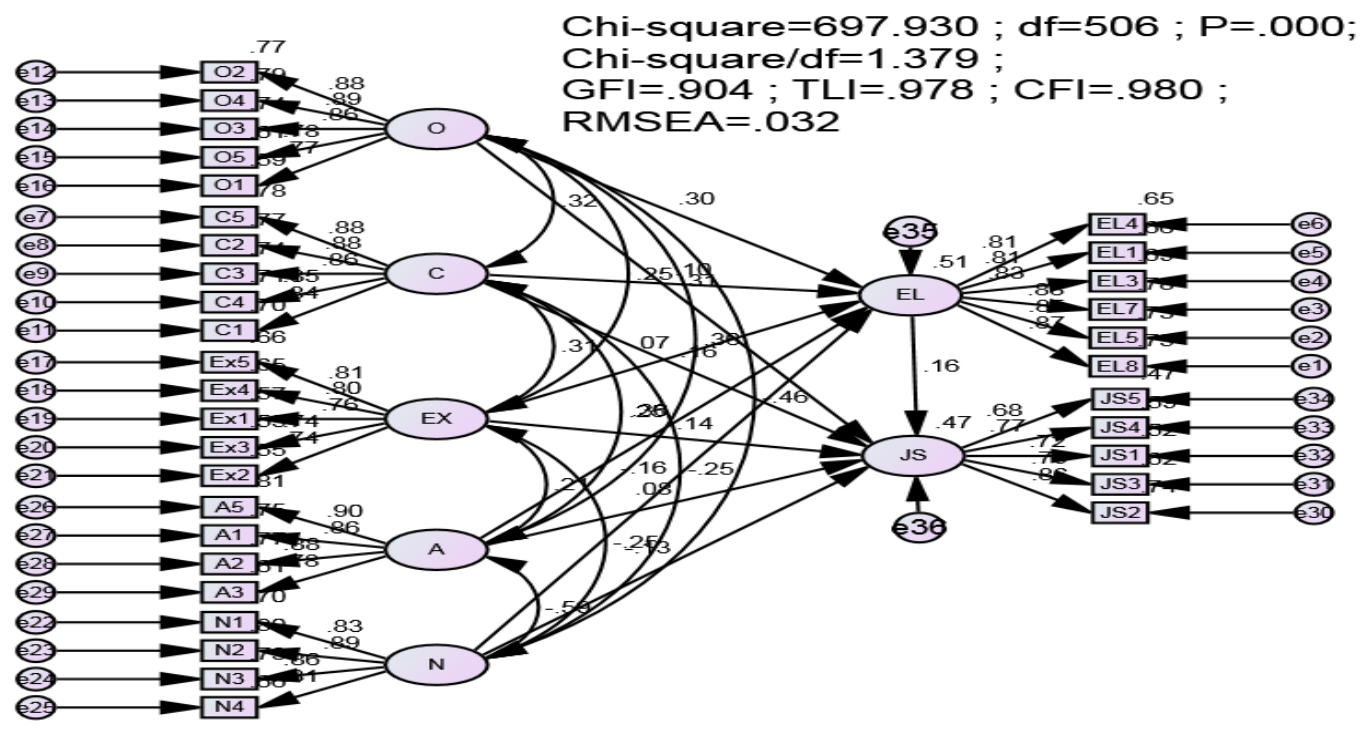

Hình 3: Kết quả SEM (chuẩn hóa) của mô hình nghiên cứu

Kết quả phân tích mô hình SEM chuẩn hóa theo bảng 3 cho thấy có 2 giả thuyết bị loại $\mathrm{H} 3$ có giá trị $\mathrm{p}$ $=0,149, \mathrm{H} 8$ có giá trị $\mathrm{p}-0,209$ đều lớn hơn 0,05 , do đó 2 giả thuyết này sẽ bị loại.

Bảng 2: Bảng hệ số hồi quy của mô hình nghiên cưu

\begin{tabular}{|c|c|c|c|c|c|c|c|}
\hline & & Quan hệ & Hệ số & S.E. & C.R. & $\mathbf{P}$ & $\begin{array}{c}\text { Kết luận giả } \\
\text { thuyết }\end{array}$ \\
\hline EL & $\begin{array}{l}<-- \\
\end{array}$ & $\mathrm{O}$ & ,255 & ,043 & 5,973 & $* * *$ & Chấp nhận \\
\hline EL & $<---$ & $\mathrm{C}$ & ,085 & ,038 & 2,248 & ,025 & Chấp nhận \\
\hline EL & $<---$ & EX & 055 & ,038 & 1,444 & , 149 & Bác bỏ \\
\hline EL & $<---$ & A & ,345 & ,053 & 6,563 & $* * *$ & Chấp nhận \\
\hline EL & $<---$ & $\mathrm{N}$ &,- 169 & 061 & $-2,761$ & ,006 & Chấp nhận \\
\hline JS & $<---$ & EL &, 173 & ,072 & 2,401 & ,016 & Chấp nhận \\
\hline JS & $<---$ & $\mathrm{N}$ &,- 151 & ,073 & $-2,054$ & ,040 & Chấp nhận \\
\hline JS & $<---$ & A & ,083 & ,066 & 1,257 & 209 & Bác bỏ \\
\hline JS & $<---$ & $\mathrm{EX}$ & , 128 & ,046 & 2,803 & ,005 & Chấp nhận \\
\hline JS & $<---$ & $\mathrm{C}$ & , 146 & ,045 & 3,212 & ,001 & Chấp nhận \\
\hline JS & $<---$ & $\mathrm{O}$ & ,288 & 054 & 5,342 & $* * *$ & Chấp nhận \\
\hline
\end{tabular}

Nguồn: kết quả phân tích của tác giả

\section{THẢO LUẬN KẾT QUẢ NGHIÊN CỬU VÀ HÀM Ý QUẢN TRI}

\subsection{Thảo luận kết quả nghiên cứu}

Kết quả nghiên cứu cho thấy rằng, sẵn sàng trải nghiệm, tận tâm và dễ chịu có mối quan hệ tích cực với sự lãnh đạo đạo đức; tâm lý bất ổn có một mối quan hệ tiêu cực với lãnh đạo đạo đức trong khi hướng ngoại không bị ảnh hưởng. Kết quả của nghiên cứu cho thấy sự tương ứng nhiều với những phát hiện của Özbağ (2016). Trong khi nghiên cứu của Boakye và Gyambrah (2017) cho thấy chỉ có hai yếu tố tận tâm và hướng ngoại có ảnh hưởng tích cực đến lãnh đạo đạo đức và tâm lý bất ổn có ảnh hưởng tiêu cực, còn hai yếu tố sẵn sàng trải nghiệm và dễ chịu không có ảnh hưởng gì đến lãnh đạo đạo đức. 
Kết quả nghiên cứu trên còn cho thấy các yếu tố sẵn sàng trải nghiệm, tận tâm và hướng ngoại có ảnh hưởng tích cực đến sự hài lòng trong công việc và ngược lại yếu tố tâm lý bất ổn có ảnh hưởng tiêu cực. Chỉ có duy nhất yếu tố dễ chịu không có ảnh hưởng đến sự hài lòng trong công việc giống kết quả nghiên cứu của Bui (2017). Trong khi nghiên cứu của Yang và Hwang (2014) chỉ có yếu tố hướng ngoại tác động đến sự hài lòng trong công việc, đều này chứng tỏ môi trường văn hóa khác nhau thì kết quả nghiên cứu sẽ khác nhau.

Về mặt thực tiễn, kết quả nghiên cứu nhằm gợi ý cho nhà lãnh đạo quản lý và nhà tuyển dụng nắm bắt được đặc điểm tính cách của nhân viên nhằm đáp ứng mang lại sự hài lòng trong công việc để đạt được kết quả làm việc tốt nhất.

\subsection{Hàm ý quản trị}

Nghiên cứu giúp các nhà lãnh đạo, nhà quản trị thấy được tác động của đặc điểm tính cách Big - Five và lãnh đạo đạo đức đến sự hài lòng trong công việc của nhân viên siêu thị. Từ đó các nhà lãnh đạo kinh doanh hiểu được giá trị của đạo đức, biết được đặc điểm tính cách nào phù hợp với chính mình và với nhân viên mang đến sự hài lòng trong công việc của nhân viên. Hãy trở thành một nhà lãnh đạo đạo đức để quản lý doanh nghiệp thành công hơn. Ngay cả chính nhân viên cũng phải biết xác định đặc điểm tính cách của mình có phù hợp với công việc này không để biết điều chỉnh cho thỏa nhu cầu. Và tìm một môi trường làm việc tốt nơi có nhà lãnh đạo biết quan tâm đến lợi ích chung của nhân viên và cộng đồng mang lại sự hài lòng cao trong công việc.

Đặc điểm tính cách đóng một vai trò quan trọng trong việc xác định sự hài lòng trong công việc, và do đó các nhà quản trị nguồn nhân lực phải quan tâm chú ý đến các thuộc tính này trong quá trình tuyển dụng, bố trí sắp xếp công việc phù hợp với tính cách của mỗi người nhằm đem lại sự hài lòng trong công việc, gắn kết nhân viên làm việc lâu dài, giúp cho các hệ thống siêu thị hoạt động tốt hơn. Hơn nữa, nghiên cứu còn giúp cho nhân viên có những định hướng nghề nghiệp phù hợp với tính cách cá nhân.

Mặc dù nghiên cứu tính dễ chịu không ảnh hưởng đến sự hài lòng nhưng đặc tính cũng quan trọng cần được xem xét trong các nghiên cứu tiếp theo.

\section{HẠN CHẾ CỦA NGHIÊN CỨU}

Thứ nhất, nghiên cứu chỉ khảo sát một số nhân viên tại một số siêu thị lớn tại Tp. HCM, chư mang tính đại diện cao. Thứ hai, câu hỏi khảo sát và mô hình nghiên cứu dự trên các nghiên cứu từ phương Tây, chưa phù hợp nhiều với văn hóa tại Việt Nam. Thứ ba, nghiên cứu chỉ dùng lại ở các siêu thị, chưa mở ra nhiều ngành nghề lĩnh vực khác. Dó đó, định hướng sắp tới của nghiên cứu là mở rộng ra các lĩnh vực, ngành nghề khác.

\section{Các chữ viết tắt:}

$\begin{array}{ll}\text { ELS } & \text { Ethical leadership scale } \\ \mathrm{O} & \text { Openness to Experience } \\ \mathrm{C} & \text { Conscientiousness } \\ \mathrm{E} & \text { Extraversion } \\ \mathrm{A} & \text { Agreeableness } \\ \mathrm{N} & \text { Neuroticism } \\ \text { EL } & \text { Ethical leadership } \\ \text { JS } & \text { Job Satisfaction } \\ \text { EFA } & \text { Exploratory factor analysis } \\ \text { CFA } & \text { Confirmatory factor analysis } \\ \text { KMO } & \text { Kaiser-Meyer-Olkin measure of sampling adequacy }\end{array}$




\section{TÀI LIỆ THAM KHẢO}

[1] P. J. Corr and G. Matthews, The Cambridge handbook of personality psychology. Cambridge, U.K: 1, 2009.

[2] R. R. McCrae and O. P. John, “An Introduction to the Five-Factor Model and Its Applications," J. Pers., vol. 60, no. 2, pp. 175-215, 1992.

[3] M. E. Brown, L. K. Treviño, and D. A. Harrison, "Ethical leadership: A social learning perspective for construct development and testing," Organ. Behav. Hum. Decis. Process., vol. 97, no. 2, pp. 117-134, 2005.

[4] H. Tutar, M. Altinöz, and D. Çakiroğlu, "Is ethical leadership and strategic leadership a dilemma? A descriptive survey," Procedia - Soc. Behav. Sci., vol. 24, no. January, pp. 1378-1388, 2011.

[5] T. A. Wright and J. C. Quick, “The role of character in ethical leadership research,” Leadersh. Q., vol. 22, no. 5, pp. 975-978, 2011.

[6] K. Kalshoven, D. N. Den Hartog, and A. H. B. de Hoogh, "Ethical Leader Behavior and Big Five Factors of Personality," J. Bus. Ethics, vol. 100, no. 2, pp. 349-366, 2011.

[7] R. L. Dhar, "Ethical leadership and its impact on service innovative behavior: The role of LMX and job autonomy," Tour. Manag., vol. 57, pp. 139-148, 2016.

[8] R. Hoppock and S. Splegler, Job Satisfaction (Harper). New York, 1953, 1935.

[9] E. A. Locke, The Nature and Causes of Job Satisfaction. Chicago: M. D. Handbook of Industrial and Organizational Psychology: R. McNally, Ed., 1976.

[10] J. Greenberg and R. A. Baron, Behavior in Organizations, 9th ed. Pearson Prentice Hall, 2008.

[11] E. Pitaloka and I. P. Sofia, "The Affect of Work Environment, Job Satisfaction, Organisation Commitment on OCB of Internal Auditors," Int. J. Bus. Econ. Law, vol. 5, no. 2, pp. 10-18, 2014.

[12] J. Hanaysha, "Determinants of Job Satisfaction in Higher Education Sector: Empirical Insights from Malaysia," Int. J. Hum. Resour. Stud., vol. 6, no. 1, p. 129, 2016.

[13] J. M. Digman, "Personality structure Emergence of the five-factor model," Annu. Rev. Psychol., vol. 41, pp. 417-440, 1990.

[14] G. K. Özbağ, “The Role of Personality in Leadership: Five Factor Personality Traits and Ethical Leadership,” Procedia - Soc. Behav. Sci., 2016.

[15] I. Boakye and M. Gyambrah, "Effect of Personality Trait on Ethical Leadership in Selected Basic Schools in Ghana,” Int. J. Sci. Res. Publ., vol. 7, no. 6, pp. 654-665, 2017.

[16] P. Naiyananont and T. Smuthranond, "Kasetsart Journal of Social Sciences Relationships between ethical climate , political behavior, ethical leadership, and job satisfaction of operational of fi cers in a wholesale company , Bangkok Metropolitan region,” Kasetsart J. Soc. Sci., pp. 1-7, 2016.

[17] C. Yang and M. Hwang, "Personality traits and simultaneous reciprocal influences between job performance and job satisfaction," Emerald Gr. Publ. Ltd., vol. 8, pp. 6-26, 2014.

[18] H. T. Bui, "Big Five personality traits and job satisfaction,” J. Gen. Manag., vol. 42, no. 3, pp. 21-30, 2017.

[19] F. O. Walumbwa and J. Schaubroeck, "Leader personality traits and employee voice behavior: Mediating roles of ethical leadership and work group psychological safety," ournal Appl. Psychol., vol. 94, no. 5, pp. 1275-1286, 2009.

[20] N. Jones, C. Hill, and C. Henn, "Personality and job satisfaction: Their role in work-related psychological well-being," J. Psychol. Africa, vol. 25, no. 4, pp. 297-304, 2015.

[21] Q. Zhai, M. Willis, B. O'Shea, Y. Zhai, and Y. Yang, "Big Five personality traits, job satisfaction and subjective wellbeing in China," Int. J. Psychol., vol. 48, no. 6, pp. 1099-1108, 2013.

[22] K. H. Foulkrod, C. Field, and C. V. R. Brown, "Trauma surgeon personality and job satisfaction: Results from a national survey," Am. Surg., vol. 76, no. 4, pp. 422-427, 2010.

[23] V. Bruk-Lee, H. A. Khoury, A. E. Nixon, A. Goh, and P. E. Spector, "Replicating and extending past 


\section{TÁC ĐộNG CỦA ĐẶC ĐIỂM TÍNH CÁCH BIG - FIVE VÀ LÃNH ĐẠO ĐẠO ĐỨC \\ TRONG CÔNG VIÊC, NGHIÊN CÚU TRƯÒ̀NG HOOP TRONG \\ CÁC HỆ THỐNG SIÊU THỊ LỚN TẠI THÀNH PHỐ HỒ CHÍ MINH.}

personality/job satisfaction meta-analyses," Hum. Perform., vol. 22, no. 2, pp. 156-189, 2009.

[24] P. T. Van den Berg and J. A. Feij, "Complex Relationships among Personality Traits, Job Characteristics, and Work Behaviors,” Int. J. Sel. Assess., vol. 11, no. 4, pp. 326-339, 2003.

[25] L. Yates, "Exploring the Relationship of Ethical Leadership with Job Satisfaction, Organizational Commitment, and Organizational Citizenship Behavior," J. Values-Based Leadersh., vol. 7, no. 1, pp. 1-15, 2014.

[26] M. J. Neubert, D. S. Carlson, K. M. Kacmar, J. A. Roberts, and L. B. Chonko, "The virtuous influence of ethical leadership behavior: Evidence from the field,” J. Bus. Ethics, vol. 90, no. 2, pp. 157-170, 2009.

[27] J. F. Hair, R. E. Anderson, R. L. Tatham, and W. C. Black, Multivariate data analysis with readings. PrenticeHall, International, Inc, 2006.

[28] I. H. Nunnally, JC. \& Bernstein, Psychometric Theory, Third edit. New York: Me Graw-Hill., 1994.

[29] W. C. Hair J.F, Anderson, R.E., Tatham, R.L., \& Black, Multivariate data analysis with readings, Fifth edit. New Jersey: Pearson Prentice Hall, 1998.

[30] J. B. E. M. Steenkamp and H. C. M. Van Trijp, “The use of lisrel in validating marketing constructs,” Int. J. Res. Mark., vol. 8, no. 4, pp. 283-299, 1991.

[31] J. Carmines, E. \& McIver, Analyzing models with unobserved variables: analusis of covariance tructures. Beverly Hills, CA, 1981.

Ngày nhận bài: 20/08/2019

Ngày chấp nhận đăng: 28/11/2019 